\title{
A Wavelength and Converter Assignment Scheme Using Converter Usage History in Wavelength-Routed Networks
}

\author{
Yukinobu Fukushima, Takahiro Ooishi and Tokumi Yokohira \\ The Graduate School of Natural Science and Technology, Okayama University, \\ 3-1-1 Tsushima-naka, Kita-ku, Okayama-city, 700-8530 Japan \\ fukusimadokayama-u.ac.jp
}

\begin{abstract}
In wavelength-routed networks, wavelength conversion improves lightpath blocking probability by eliminating the wavelength-continuity constraint. Because wavelength converters remain expensive in the near future, we need a wavelength and converter assignment scheme that decreases blocking probability with a limited number of converters. In this paper, we propose a wavelength and converter assignment scheme for decreasing blocking probability. Our scheme avoids contention among multiple lightpath requests by making each node-pair to perform wavelength conversion at different intermediate nodes with more idle converters. Simulation results show that 1) our scheme decreases blocking probability by about between $44 \%$ and $83 \%$ and 2) our scheme needs one or two fewer wavelength converters per node to achieve near-optimal blocking probability compared with conventional schemes.
\end{abstract}

\section{KEYWORDS}

WDM, Wavelength and converter assignment problem, Wavelength conversion

\section{INTRODUCTION}

With the population of video streaming [1], [2] on the Internet, there is an increasing need for bandwidth. Wavelength-Division Multiplexing (WDM) provides huge bandwidth by allowing simultaneous data transmission on multiplexed wavelengths on a single fiber. Among several network architectures for WDM network, wavelength-routed network is believed to be the most promising architecture [3]-[6]. In wavelength-routed networks, access networks communicate with each other via all-optical connections called lightpaths.

Performance of wavelength-routed network (i.e., blocking probability of lightpaths) depends on routing and wavelength assignment (RWA) algorithm, which determines a route and an assigned wavelength of each lightpath. RWA can be performed either in a centralized or distributed manner. Because centralized network control can not cope with the rapid growth of WDM networks because of the lack of scalability, distributed network control is more suitable [7]-[9]. In conventional research for RWA, RWA is divided into a routing sub-problem and a wavelength assignment sub-problem because RWA is time-consuming [10]. This paper focuses on a wavelength-assignment sub-problem.

Wavelength conversion improves lightpath blocking probability by eliminating the wavelength continuity constraint (i.e., the constraint that the same wavelength must be assigned to a lightpath on links along a route) [11]-[14]. However, because wavelength converter cost remains expensive in the near future, the number of wavelength converters deployed in the network is limited. 
Therefore, we need to realize as low blocking probability as possible with limited number of wavelength converters.

Most of the conventional wavelength-assignment algorithms (e.g., First-Fit (FF), Most-Used (MU) and Least-Used (LU)) only perform wavelength assignment and do not consider converter assignment. As a result, they tend to assign more converters than needed in order to establish a lightpath, and consequently, the more forthcoming lightpaths are blocked because of wavelength continuity constraint. Some conventional algorithms (First-Longest lambda-Run (FLR) [15] and LEast Converter first (LEC) [16]) consider both wavelength and converter assignment. They try to decrease lightpath blocking probability by minimizing the number of converters assigned to a lightpath. However, in order to decrease blocking probability, it is more important to avoid multiple node-pairs from reserving the same wavelength converter at the same time.

In this paper, we propose a wavelength and converter assignment scheme for decreasing blocking probability. Our scheme avoids contention of lightpath requests among multiple node-pairs by making each node-pair to perform wavelength conversion at different intermediate nodes. Each node-pair can realize this by selecting its preferred converter based on statistical information learned from wavelength converter usage history. In addition, our proposed scheme tries to perform wavelength conversion at intermediate nodes with more idle converters in order to keep as many wavelength conversion nodes as possible available. Key idea behind our scheme is based on Priority-based Wavelength Assignment (PWA) [17], which only considers wavelength assignment in optical burst switching (OBS) networks [18]. The PWA avoids multiple node-pairs from reserving the same wavelength using statistical information learned from wavelength priority database.

The paper is organized as follows. In section 2, we present the network architecture and routing/signaling protocol that we adopt, and we explain the conventional wavelength and converter assignment schemes. We propose our scheme using converter usage history in section 3 . Then, we show our evaluation results in section 4 . Section 5 concludes this paper.

\section{Wavelength and Converter Assignment in Wavelength- ROUTED NETWORKS}

\subsection{Network Architecture}

Figure 1 depicts architecture of wavelength-routed networks. It consists of edge/core wavelength routing nodes interconnected by fibers. Wavelength routing nodes exchange link state information with a routing protocol such as OSPF-TE [19] in order to obtain network topology and resource usage information of each link. When an access network communicates with other access network, it first determines a route of a lightpath based on topology and resource usage information. Then, it sets up the lightpath with a signaling protocol such as RSVP-TE [20]. We assume that the destination-initiated reservation (DIR) [21] is adopted for resource reservation method. In the DIR, lightpath request message from source to destination only collects link state information along the route. Then, the destination node selects an appropriate wavelength and converter, and sends a reservation request to reserve the wavelength along the route.

Figure 2 shows a node architecture that we assume in this research. It consists of demultiplexers (DEMUX), Optical Cross-Connects (OXC), multiplexers (MUX) and wavelength converters (WC). When a node relays a wavelength for establishment of a lightpath, a DEMUX first demultiplexes an input signal into each wavelength. Then, an OXC switches each wavelength to 
an appropriate output port. Finally a MUX multiplexes wavelengths into an output signal. When the wavelength same as an input wavelength is not idle on an output fiber, the input wavelength

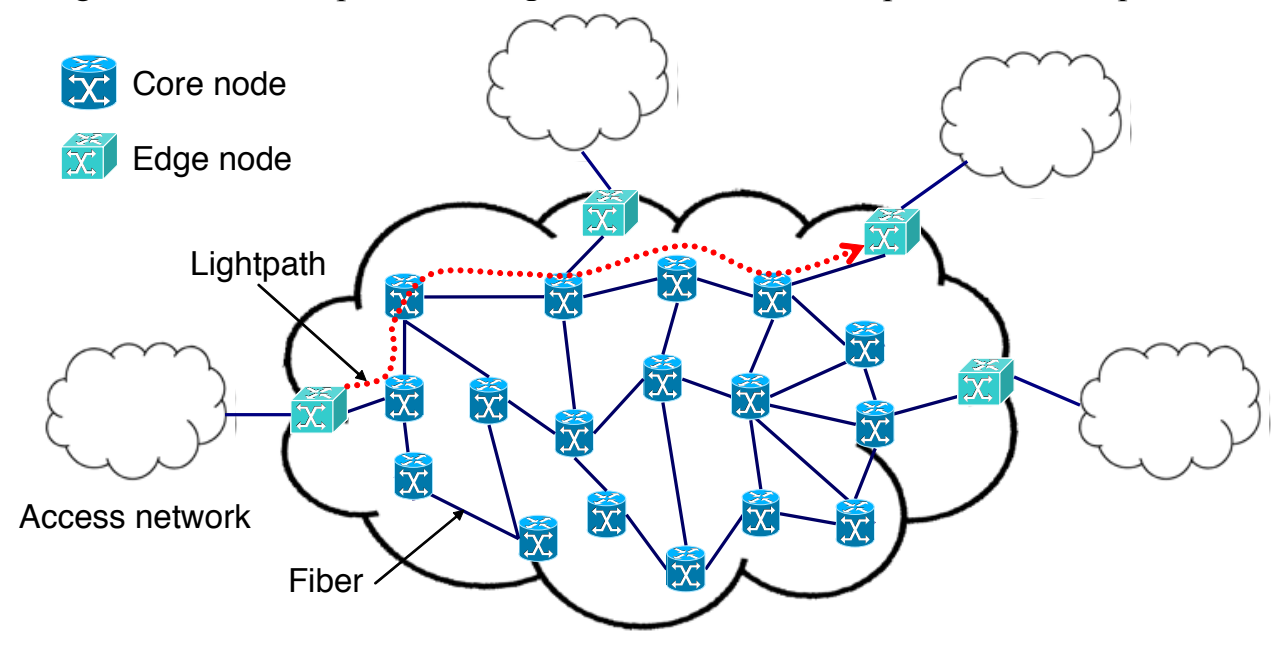

Wavelength-routed network

Figure 1. Wavelength-routed network

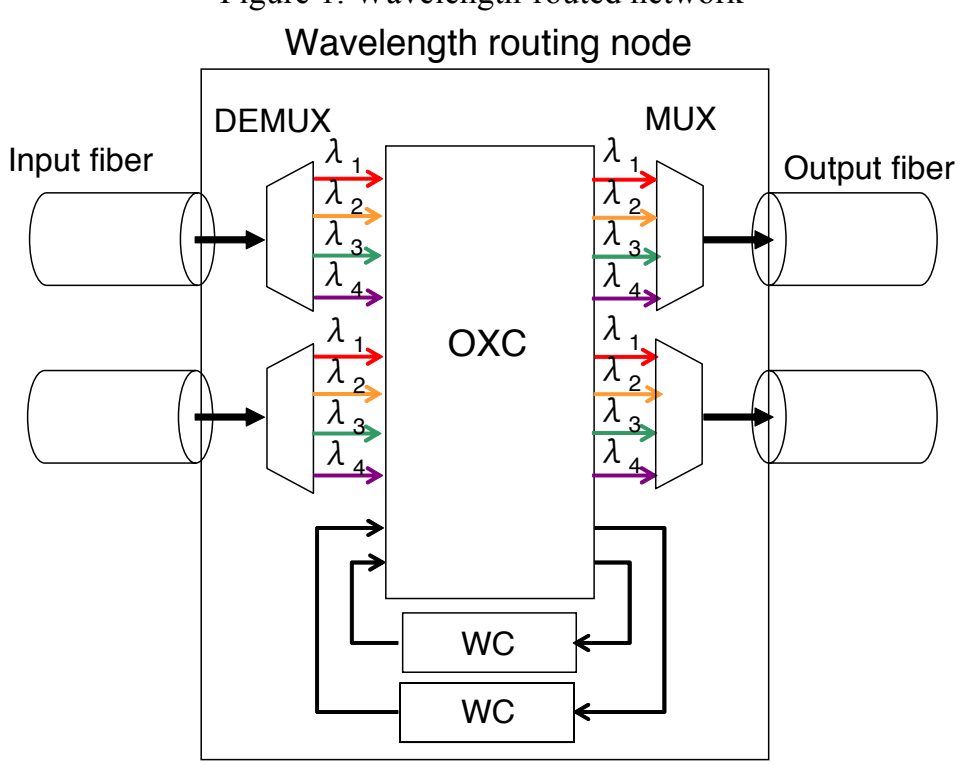

Figure 2. Node architecture

is switched to a wavelength converter and converted to another wavelength idle on an output fiber.

We assume that wavelength converters are deployed on nodes in a trunk-type basis [22]. In the trunk-type, wavelength converters are shared among input ports. The input port that actually needs wavelength conversion is switched to an output port with a full wavelength converter. As a result, the number of wavelength converters deployed is reduced.

\subsection{Wavelength and Converter Assignment Problem}

The wavelength and converter assignment problem that we tackle in this paper is expressed as follows: given 1) a route of a requested lightpath and 2) link-state information of links on the route, determine wavelengths and converters assigned to the lightpath so that the total blocking probability is minimized. 
International Journal of Computer Networks \& Communications (IJCNC) Vol.5, No.4, July 2013

\subsection{Conventional Wavelength and Converter Assignment Schemes}

\subsubsection{FLR (First Longest-Lambda Run)}

The FLR [15] tries to decrease blocking probability by minimizing the number of converters assigned to a lightpath. The FLR uses the concept of lambda-run. A lambda-run is a sequence of the same wavelengths that are idle on successive links along a route. In addition, a lambda-run satisfies the following conditions: 1) originating from the source node of a lightpath or an intermediate node with at least one idle converter, 2) terminating at the destination node of a lightpath or an intermediate node with at least one idle converter, and 3) being as long as possible.

The FLR repeatedly selects the longest lambda-run from the source node to the destination node. At the terminal of each lambda-run, it performs wavelength conversion. If a set of lambda-runs from the source to the destination is found, the lightpath is successfully set up, otherwise, blocked. In [15], it is proven that this simple policy leads to minimizing the number of wavelength conversions in setting up a lightpath.

\subsubsection{LEC (LEast Converter first)}

The LEC [16] tries to decrease blocking probability by minimizing the number of converters assigned to a lightpath as well as the FLR. The LEC uses an auxiliary graph that represents wavelength and converter usage information on a route of a lightpath.

When the LEC sets up a lightpath, it first constructs an auxiliary graph based on link-state information on the route. Then, the LEC assigns a constant cost $f$ to an edge corresponding to an idle wavelength on a link, an infinity cost to an edge corresponding to an used wavelength on a link, and a constant cost $g$ to an edge corresponding to performing wavelength conversion on an intermediate node. Note that wavelength conversion cost $g$ is larger than cost of any path without wavelength conversion (i.e., $g>n f$, where $n$ is the hop count of the route). After that, the LEC computes the minimum cost path from source to destination with Dijkstra algorithm on the graph. If such a path is obtained, LEC reserves the selected wavelengths and converters and successfully sets up a lightpath. Otherwise, the LEC refuses the request.

\section{Wavelength and Converter Assignment Scheme Using CONVERTER USAGE HiSTORY}

We propose a wavelength and converter assignment scheme that tries to decrease lightpath blocking probability. Our scheme avoids contention among multiple lightpath requests by making each node-pair to perform wavelength conversion at different intermediate nodes with more idle converters.

Our proposed scheme sets up the requested lightpath with the following steps:

1) Construction of auxiliary graph based on link state information on the route

2) Calculation of wavelength conversion cost on intermediate nodes with idle converters

3) Determination of the wavelengths and converters that are assigned to the lightpath

4) Reservation of those wavelengths and converters

Given a route of a requested lightpath and link-state information on the route, our scheme first constructs an auxiliary graph. The graph represents wavelength and converter usage information 
on a route of a lightpath. Figure 4 shows an example of the auxiliary graph on a route with six nodes in Fig. 3. In the graph, a vertex corresponds to an input or output port of a node, an edge between different nodes corresponds to a wavelength, and an edge within the same node corresponds to a connection between input and output ports. In addition, the source (destination) node has a vertex corresponding to traffic-source (traffic-destination) instead of those corresponding to input (output) ports. Note that using edges connecting an input port to an output port on different wavelength means converting an input wavelength to a different one with a wavelength converter.

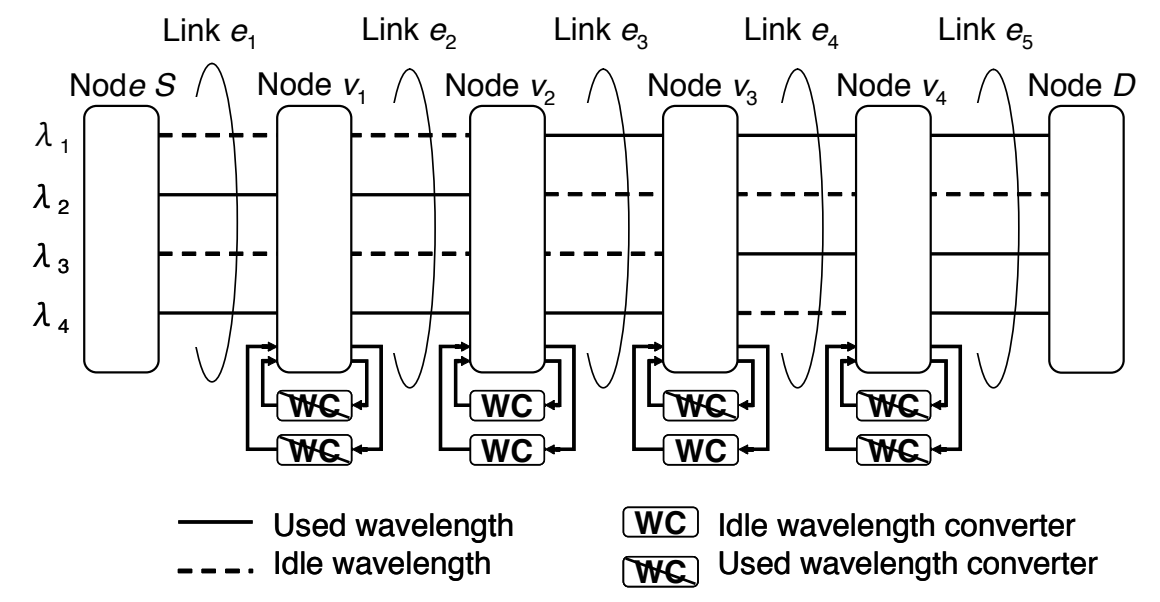

Figure 3. Route with six nodes (number of multiplexed wavelengths: 4)

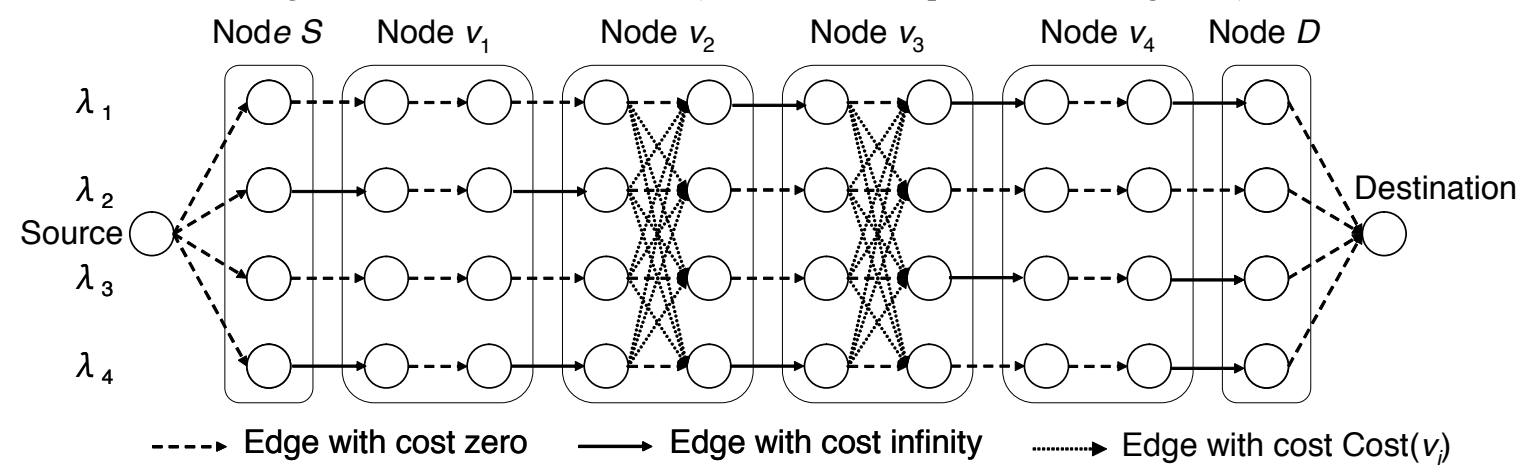

Figure 4. Auxiliary graph on a route with six nodes

Costs of edges are determined as follows. We assign zero to idle wavelengths while we assign infinity to used ones. We assign zero to connections from traffic-source to output ports, those from input ports to traffic-destination and those from an input port to an output port on the same wavelength, while we assign wavelength conversion cost $\left(\operatorname{Cost}\left(v_{i}\right)\right)$ to connection from an input port to an output port on different wavelengths on node $v_{i}$.

We define wavelength conversion cost $\left(\operatorname{Cost}\left(v_{i}\right)\right)$ of intermediate node $v_{i}$ with idle converters as follows:

$$
\operatorname{Cost}\left(v_{i}\right)=\frac{U_{i}}{A_{i}}
$$

where $U_{i}$ is the proportion of the node-pairs that used wavelength converters on node $v_{i}$ in $M$ latest entries of wavelength converter usage history except the source-destination pair of the requested lightpath, and $A_{i}$ is the number of idle converters on node $v_{i}$. By using $U_{i}$, we expect 
that node-pairs tend to perform wavelength conversion on different intermediate nodes, and consequently, contention among them is avoided. In addition, we expect that wavelength converters are available on as many intermediate nodes as possible by using $A_{i}$.

After determining cost of each edge, our scheme performs Dijkstra algorithm on the auxiliary graph and determines idle wavelengths and converters to be assigned to the lightpath so that the sum of wavelength conversion costs is the minimum. When there are multiple wavelengths with the same wavelength conversion cost, our scheme selects the wavelength with the minimum index (i.e., First-Fit policy). If idle wavelengths and converters are found, the lightpath is successfully set up, otherwise it is blocked.

We show an example of establishing a lightpath with our proposed scheme on the route with six nodes in Fig. 3. Intermediate nodes $v_{1}, v_{2}, v_{3}$ and $v_{4}$ have zero, two, one and zero idle converters, respectively. The number of entries in wavelength converter usage history $(M)$ is three. The entry of wavelength converter usage history of intermediate nodes with idle converters are shown in Tab. 1. The wavelength converter usage history on node $v_{2}$ consists of node-pairs $(S, D),\left(v_{1}, D\right)$ and $\left(S, v_{4}\right)$. This means that the three node-pairs performed wavelength conversion on node $v_{2}$ in the past. Similarly, the wavelength converter history of node $v_{3}$ has $\left(v_{1}, D\right),\left(v_{2}, D\right)$ and $\left(v_{2}, v_{4}\right)$. In this case, $\operatorname{Cost}\left(v_{2}\right)=(2 / 3) / 2=1 / 3$, and $\operatorname{Cost}\left(v_{3}\right)=(3 / 3) / 1=1$. By applying Dijkstra algorithm, our proposed scheme selects the following wavelengths and converters with the minimum cost: $\lambda_{1}$ on links $e_{1}$ and $e_{2}$, a wavelength converter on node $v_{2}$ and $\lambda_{2}$ on links $e_{3}, e_{4}$ and $e_{5}$.

Table 1. Converter usage history of intermediate node with idle converters $(M=3)$

\begin{tabular}{|c|c|c|c|}
\hline Node & Latest & $\mathbf{2}^{\text {nd }}$ latest & $\mathbf{3}^{\text {rd }}$ latest \\
\hline$v_{2}$ & $(S, D)$ & $\left(v_{1}, D\right)$ & $\left(S, v_{4}\right)$ \\
\hline$v_{3}$ & $\left(v_{1}, D\right)$ & $\left(v_{2}, D\right)$ & $\left(v_{2}, v_{4}\right)$ \\
\hline
\end{tabular}

\section{Performance Evaluation}

\subsection{Simulation Model}

We compare our proposed scheme with conventional schemes, FLR and LEC. As network model, we use 16-node mesh network (Fig. 5) and 14-node NSFNET (Fig. 6). The number of wavelengths multiplexed on each fiber is set to 16 . We deploy identical number of wavelength converters on each node. The number of lightpath requests follows Poisson distribution with rate $\lambda$. Lightpath holding time follows exponential distribution with rate $1 / \mu$. We define traffic load as $\lambda / \mu$ [Erlang]. We adopt least load routing (LLR) that selects the route with the most idle wavelengths among candidate routes. In LLR, the number $(P)$ of candidate routes is set to four or eight. We set wavelength usage history size $(M)$ to ten because our simulation results for various wavelength usage history sizes confirmed that size more than ten achieves almost the same lightpath blocking probability. 


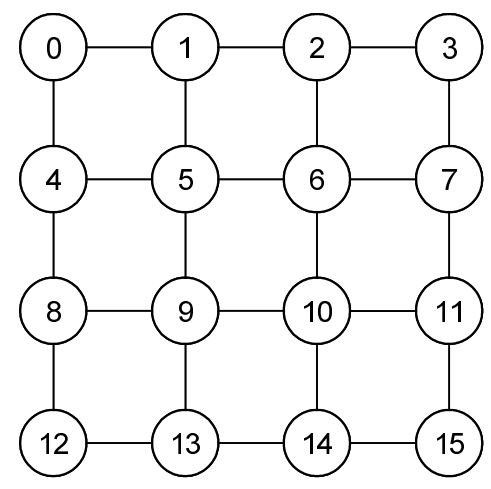

Figure 5. 16-node mesh network

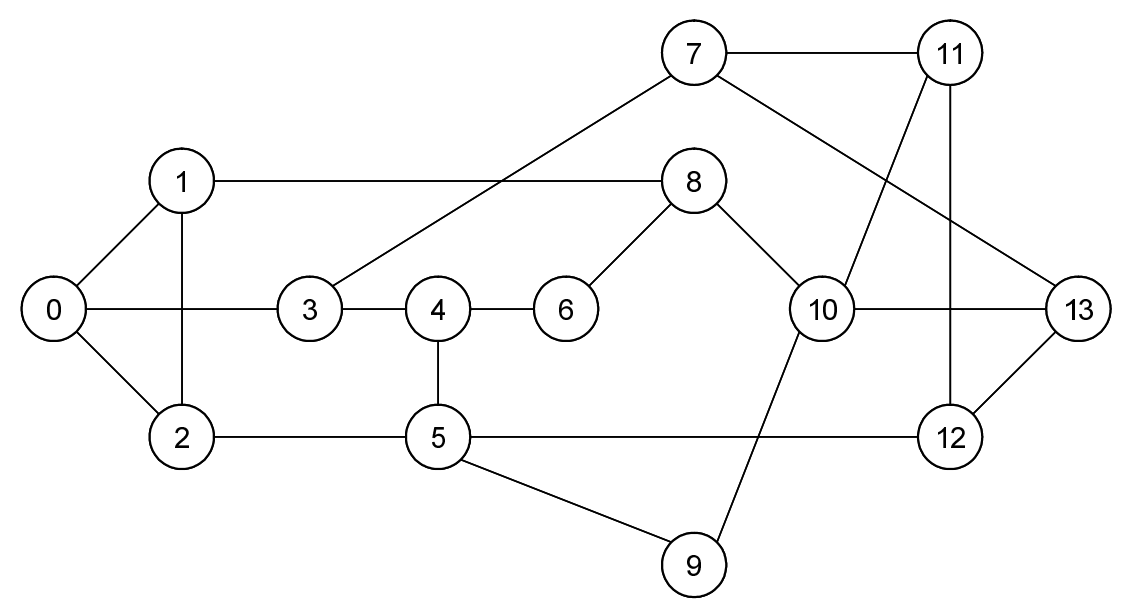

Figure 6. 14-node NSFNET

\subsection{Evaluation Results}

Figures 7 and 8 show lightpath blocking probability as a function of the number of converters per node in mesh network. Our proposed scheme achieves lower blocking probability than FLR and LEC when the number of converters per node ranges between 2 and 10. Compared with FLR and LEC, out proposed scheme decreases blocking probability by about $44 \%$ for $P=4$ and by about $83 \%$ for $P=8$ at the maximum.

On the other hand, our proposed scheme shows almost the same blocking probability as the conventional schemes when the number of converters per node is too small (e.g., less or equal to 2 ) or too large (e.g., greater or equal to 10). This is explained as follows. When the number of converters per node is too small, we have little chance to perform wavelength conversion and the performance improvement by wavelength conversion is small. Thus, there is little difference in blocking probability among different schemes. When the number of converters per node is too large, the wavelength continuity constraint is eliminated by the enough conversion capacity and blockings are only caused by lack of wavelength resource. Therefore, in this case, there is also little difference in blocking probability among different schemes. 
International Journal of Computer Networks \& Communications (IJCNC) Vol.5, No.4, July 2013

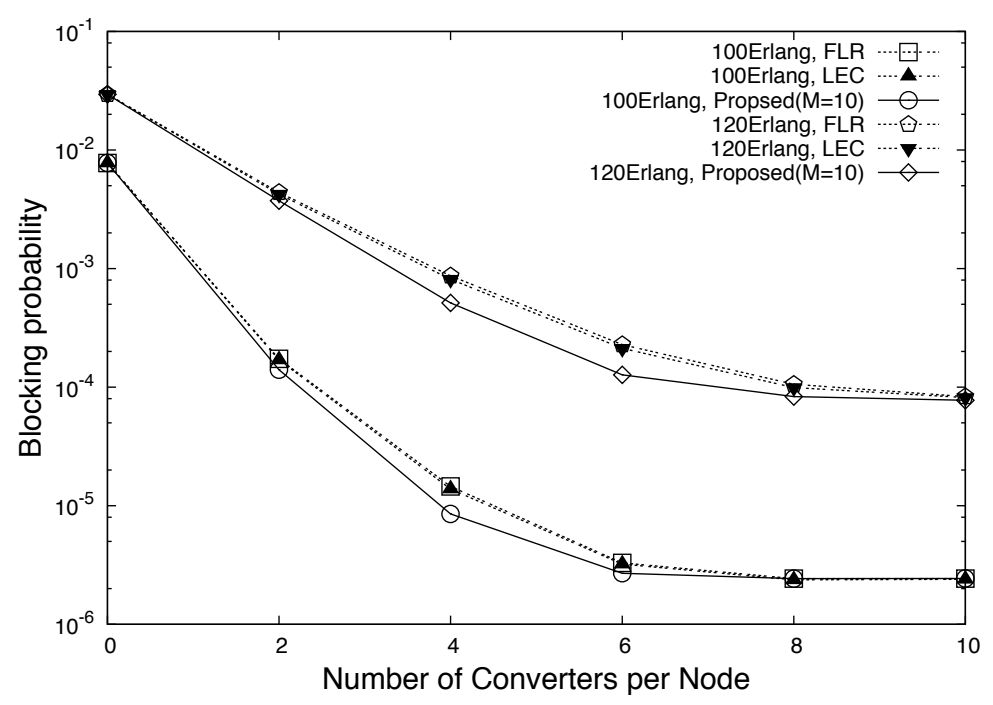

Figure 7. Lightpath blocking probability (mesh network, $P=4$ )

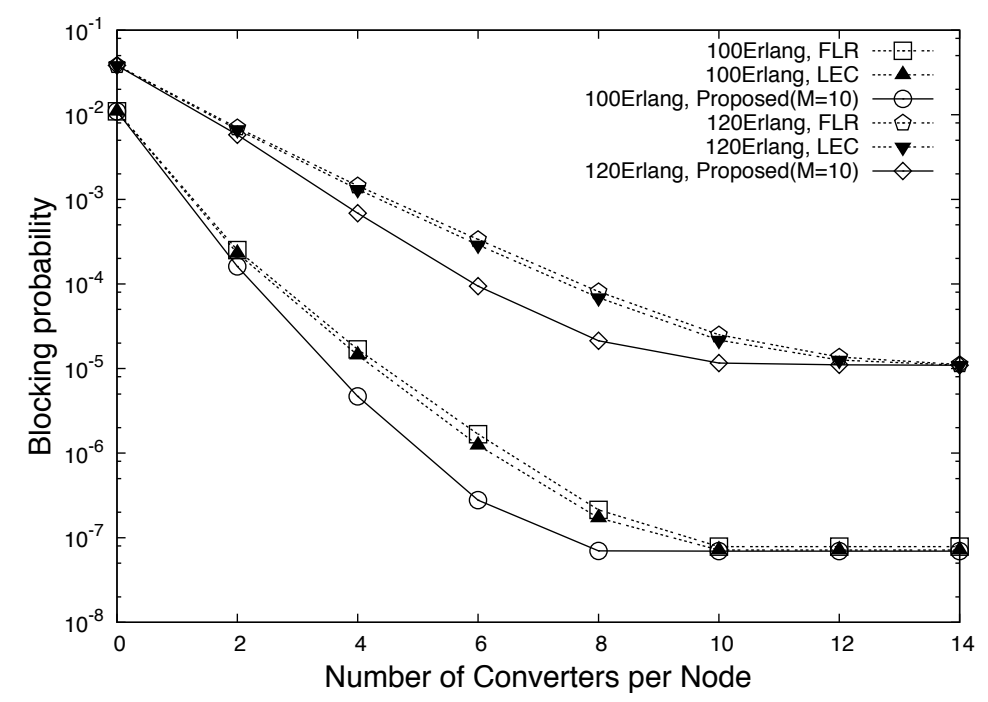

Figure 8. Lightpath blocking probability (mesh network, $P=8$ )

We next evaluate the minimum number of wavelength converters per node in order to achieve near optimal blocking performance. We regard the minimum number $(X)$ that satisfies the following equation [23] as the minimum number of converters.

$$
\frac{P_{B}(X)-P_{B}(\infty)}{P_{B}(0)-P_{B}(\infty)}=\varepsilon
$$

$P_{B}(0) / P_{B}(\infty) / P_{B}(X)$ are blocking probabilities when no/infinite/ $X$ converters are deployed on each node, respectively. We set 0.001 to $\varepsilon$.

Table 2 depicts $X$ for each scheme. For different traffic load and the number of candidate routes, our proposed scheme shows one or two fewer converters per node than FLR and LEC. This means that, from the viewpoint of the whole network, our proposed scheme can decrease the total number of converters by between sixteen and thirty-two. 
Table 2. Minimum number of converters per node in order to achieve near optimal blocking probability (mesh network)

\begin{tabular}{|c|c|c|c|c|}
\hline & \multicolumn{2}{|c|}{100 Erlang } & \multicolumn{2}{c|}{120 Erlang } \\
\hline & $P=4$ & $P=8$ & $P=4$ & $P=8$ \\
\hline FLR & 5 & 5 & 8 & 9 \\
\hline LEC & 5 & 5 & 8 & 9 \\
\hline Proposed & 4 & 4 & 7 & 7 \\
\hline
\end{tabular}

Figures 9 and 10 show lightpath blocking probability as a function of the number of converters per node in NSFNET. Similarly to those in mesh network, our proposed scheme achieves lower blocking probability than FLR and LEC when the number of converters per node ranges between 2 and 10. Compared with FLR and LEC, out proposed scheme decreases blocking probability by about $57 \%$ for $P=4$ and by about $60 \%$ for $P=8$ at the maximum.

Table 3 summarizes the minimum number of converters per node to achieve near-optimal blocking probability for each scheme. Our proposed scheme shows one fewer converters per node than FLR while it shows the same number of converters per node as LEC for 100 Erlang and one fewer converters than LEC for 120 Erlang.

\section{Conclusions}

In this paper, we have proposed a wavelength and converter assignment scheme in wavelengthrouted networks. Our scheme avoids contention among multiple lightpath requests by making each node-pair to perform wavelength conversion at different intermediate nodes with more idle converters. Our scheme realize this by using statistical information learned from wavelength converter usage history and the number of idle converters on intermediate nodes. Simulation results show that our scheme decreases blocking probability by about between $44 \%$ and $83 \%$ and our scheme needs one or two fewer wavelength converters per node to achieve near-optimal blocking probability compared with conventional schemes. We conclude that wavelength converter usage history is useful for distributing wavelength conversion point of node-pairs and decreasing lightpath blocking probability.

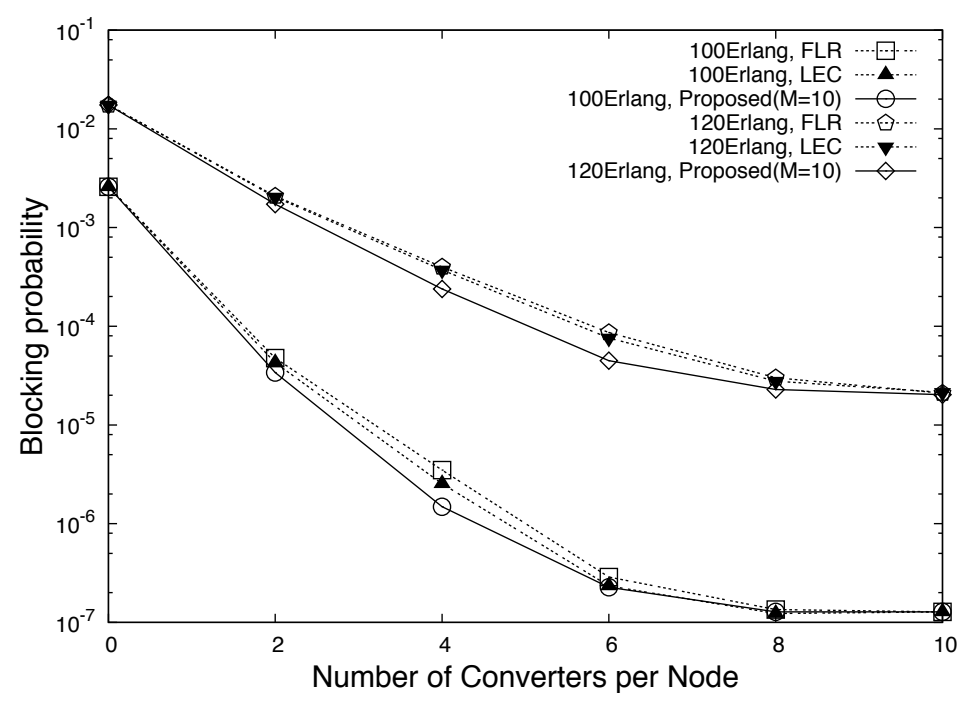

Figure 9. Lightpath blocking probability (NSFNET, $P=4)$ 
International Journal of Computer Networks \& Communications (IJCNC) Vol.5, No.4, July 2013

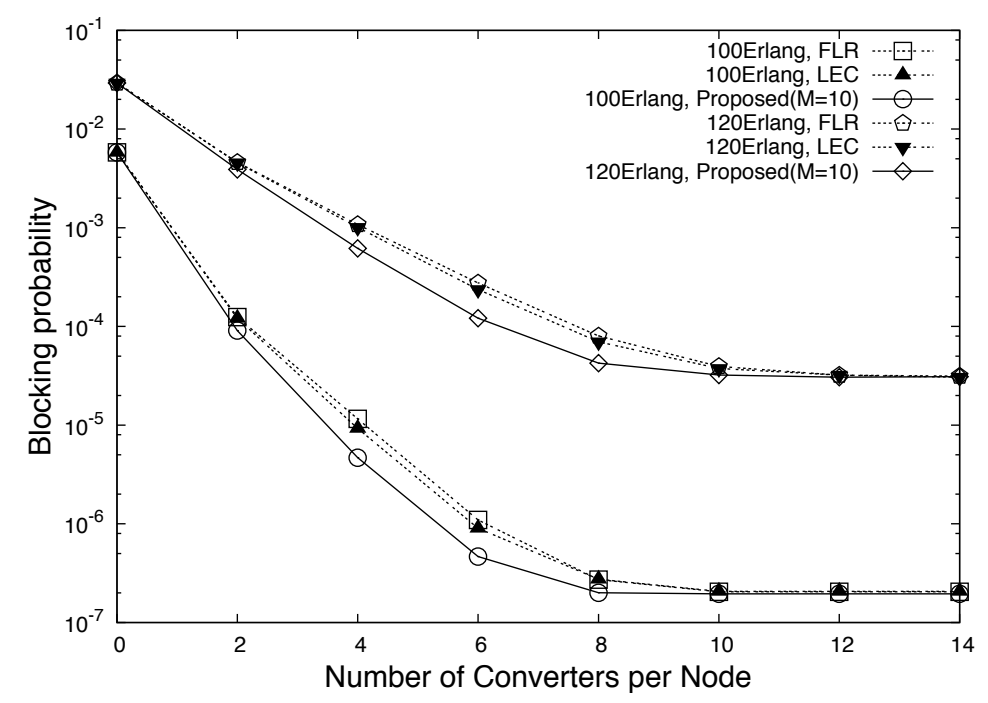

Figure 10. Lightpath blocking probability (NSFNET, $P=8$ )

Table 3. Minimum number of converters per node in order to achieve near optimal blocking probability (NSFNET)

\begin{tabular}{|c|c|c|c|c|}
\hline & \multicolumn{2}{|c|}{100 Erlang } & \multicolumn{2}{c|}{ 120 Erlang } \\
\hline & $P=4$ & $P=8$ & $P=4$ & $P=8$ \\
\hline FLR & 5 & 5 & 8 & 9 \\
\hline LEC & 4 & 4 & 8 & 9 \\
\hline Proposed & 4 & 4 & 7 & 8 \\
\hline
\end{tabular}

\section{REFERENCES}

[1] L. Favalli, M. Folli, A. Lombardo, D. Reforgiato, and G. Schembra, “A P2P platform for real-time multicast video streaming leveraging on scalable multiple descriptions to cope with bandwidth fluctuations," International Journal of Computer Networks and Communications, Vol. 3, No. 6, Nov. 2011.

[2] H. Oda, H. Hisamatsu, and H. Noborio, "Design, implementation, and evaluation of congestion control mechanism for video streaming," International Journal of Computer Networks and Communications, Vol. 3, No. 3, May. 2011.

[3] I. Chlamtac, A.Ganz, and G.Karmi, "Lightpath communications: An approach to high bandwidth optical WAN's," IEEE Transactions on Communications, vol. 40, pp. 1171-1182, July 1992.

[4] I. Chlamtac, A. Farago, and T. Zhang, "Lightpath (wavelength) routing in large WDM networks," IEEE Journal on Selected Areas in Communications, vol. 14, pp. 909-913, June 1996.

[5] B. Mukherjee, D. Banerjee, S. Ramamurthy, and A. Mukherjee, "Some principles for designing a wide-area WDM optical networks," IEEE/ACM Transactions on Networking, vol. 4, pp. 684-696, Oct. 1996.

[6] R. Ramaswami and K. N. Sivarajan, "Design of logical topologies for wavelength-routed optical networks," IEEE Journal on Selected Areas in Communications, vol. 14, pp. 840-851, June 1996.

[7] J. Zheng and H. T. Mouftah, "Distributed lightpath control based on destination routing for wavelength-routed WDM networks," in Proceedings of GLOBECOM, Nov. 2001.

[8] H. Zang, J. P. Jue, L. Sahasrabuddhe, R. Ramamurthy, and B. Mukherjee, "Dynamic lightpath establishment in wavelength-routed WDM networks," IEEE Communications Magazine, vol. 39, pp. 100-108, Sept. 2001 
International Journal of Computer Networks \& Communications (IJCNC) Vol.5, No.4, July 2013

[9] U. Bhanja, S. Mahapatra, and R. Roy, "A novel solution to the dynamic routing and wavelength assignment problem in transparent optical networks," International Journal of Computer Networks and Communications, Vol. 2, No. 2, Mar. 2010.

[10] H. Zang, J. P. Jue, and B. Mukherjee, "A review of routing and wavelength assignment approaches for wavelengthrouted optical WDM networks," Optical Networks Magazine, vol. 1, pp. 47-60, Jan. 2000.

[11] M. Azizoglu, Suresh Subramaniam, and Arun K. Somani, "Converter placement on wavelengthrouted network paths," in Proceedings of SPIE All-Optical Communication Systems: Architecture, Control, and Network Issues III, vol. 3230, pp. 265-276, Nov. 1997.

[12] H. Harai, M. Murata, and H. Miyahara, "Performance analysis of wavelength assignment policies in all-optical networks with limited-range wavelength conversion," IEEE Journal on Selected Areas in Communications, vol. 16, pp. 1051-1060, Sept. 1998.

[13] S. Subramaniam, M. Azizoglu, and A. K. Somani, "On optimal converter placement in wavelength-routed networks," IEEE/ACM Transactions on Networking, vol. 7, pp. 754-766, Oct. 1999.

[14] Y. Fukushima, H. Harai, S. Arakawa, and M. Murata, "Design of wavelength-convertible edge nodes in wavelengthrouted networks," OSA Journal of Optical Networking, vol. 5, pp. 196-209, Mar. 2006.

[15] Q. D. Ho and M. S. Lee, "Converter-aware wavelength assignment in WDM networks with limited-range conversion capability," IEICE Transaction on Communications, vol. E89-B, pp. 436-445, Feb. 2006.

[16] Y. Zang, K. Taira, H. Takagi, and S. K. Das, "Adaptive wavelength routing and assignment in optical WDM networks," Optical Networks Magazine, vol. 4, pp. 86-99, Sept./Oct. 2003.

[17] X. Wang, H. Morikawa, and T. Aoyama, "Priority-based wavelength assignment algorithm for burst switched WDM optical networks," IEICE Transactions on Communications, vol. E86-B, pp. 1508-1514, May 2003.

[18] C. Qiao and M. Yoo, "Optical burst switching (OBS) - a new paradigm for an optical Internet," Journal of High Speed Networks, vol. 8, pp. 69-84, Mar. 1999.

[19] K. Kompella and Y. Rekhter, "OSPF extensions in support of generalized MPLS," Internet Draft draft-ietf-ccampospf-gmpls-extensions-12.txt, Oct. 2003.

[20] L. Berger, "Generalized multi-protocol label switching (GMPLS) signaling resource reservation protocol-traffic engineering (RSVP-TE) extensions," IETF RFC 3473, Jan. 2003.

[21] Y. Mei and C. Qiao, "Efficient distributed control protocols for WDM all-optical networks," in Proceedings of ICCCN, pp. 150-153, Sept. 1997.

[22] E. Oki, D. Shimazaki, K. Shiomoto, N. Matsuura, and W. Imajuku, "Performance of distributedcontrolled dynamic wavelength-conversion GMPLS networks," in Proceedings of ICOCN, Nov. 2002.

[23] K. Xi, S. Arakawa, and M. Murata, "How many wavelength converters do we need?," in Proceedings of ONDM, pp. 347-358, Feb. 2005. 